\title{
Patients' and healthcare professionals' views of cancer follow-up: systematic review
}

Ruth A Lewis, Richard D Neal, Maggie Hendry, Barbara France, Nefyn H Williams, Daphne Russell, Dyfrig A Hughes, Ian Russell, Nicholas SA Stuart, David Weller and Clare Wilkinson

\section{ABSTRACT \\ Background}

Cancer follow-up places a significant burden on hospita outpatient clinics. There are increasing calls to develop alternative models of provision.

Aim

To undertake a systematic review of qualitative studies examining patients' and healthcare professionals' views about cancer follow-up.

Design of study

Systematic review.

Setting

Primary and secondary care.

Method

Comprehensive literature searches included: 19 electronic databases, online trial registries, conference proceedings, and bibliographies of included studies. Eligible studies included qualitative studies examining patients' and healthcare professionals' views of cancer follow-up. Studies of patients with any type of cancer, considered free of active disease, or no longer receiving active treatment were included. Findings were synthesised using thematic analysis.

Results

Nineteen studies were included; seven were linked to randomised controlled trials. Eight studies examined the views of healthcare professionals (four of which included GPs) and 16 examined the views of patients. Twelve descriptive themes were identified, from which 12 perceived implications for practice were derived. Most themes related to conventional follow-up in secondary care. Some views concerning other models of care were based on participants' ideas, rather than experiences.

\section{Conclusion}

Patients' main concern is recurrent disease, and they find regular follow-up, expertise of specialists, and quick access to tests reassuring. Information regarding the effectiveness of follow-up is not given to patients who also have unmet information needs, which would help them to cope and be more involved. Continuity of care, unhurried consultations, and psychosocial support are important, but sometimes lacking in secondary care. GPs are thought to be unwilling and to have insufficient time and expertise to conduct follow-up.

\section{Keywords}

long-term care; neoplasms; oncologic nursing;

outpatients; primary health care; systematic review.

\section{INTRODUCTION}

Conventional hospital-based follow-up of cancer patients places a significant burden on hospital outpatient clinics, and is of debatable value for many cancers in terms of early diagnosis of recurrence and improved survival..$^{1-6}$ Consequently, there is continued interest in the provision of follow-up or ongoing management in different and innovative ways. Models typically include primary care follow-up, ${ }^{7}$ nurse-led follow-up, ${ }^{8}$ telephone-based follow-up, and patientinitiated follow-up (or combinations of these).

As the number of cancer survivors increases, the focus of follow-up is shifting towards the management of a chronic condition. In the UK there is growing government pressure for the NHS to encourage patients with chronic conditions to take

RA Lewis, MSc, BSc, lecturer; RD Neal, PhD, FRCGP, senior clinical lecturer; $N H$ Williams, PhD, FRCGP, senior clinical lecturer; $M$ Hendry, BA Research fellow; B France, research assistant; C Wilkinson, MD, FRCGP, Department of Primary Care and Public Health, North Wales Clinical School, School of Medicine, Cardiff University, Wrexham; D Russell, PhD, senior research fellow/trial statistician, Institute for Medical and Social Care Research, Bangor University; NSA Stuart, DM, FRCP, North Wales Clinical School, Bangor University; DA Hughes, $P h D$ reader, Centre for Economics and Policy in Health, Bangor University, Bangor, Bangor. I Russell, PhD, FRCGP, FRCP(Ed), professor, School of Medicine, Swansea University, Swansea. D Weller, PhD, MRCGP, FRCP, professor, Department of General Practice, University of Edinburgh, Edinburgh.

\section{Address for correspondence}

Ruth Lewis, Cardiff University, Department of Primary Care and Public Health, School of Medicine, North Wales Clinical School, Gwenfro Building Unit 6/7, Wrexham Technology Park, Wrexham, LL13 7YP. E-mail: lewisr17@cf.ac.uk

Submitted: 3 November 2008; Editor's response: 6 January 2009; final acceptance: 6 February 2009.

() British Journal of General Practice

This is the full-length article of an abridged version published in print. Cite this article as: Br J Gen Pract 2009; DOI: 10.3399/bjgp09X453576. 
more responsibility for their own care, ${ }^{9}$ and there is some evidence from other chronic conditions, such as diabetes, that empowerment could lead to better patient outcomes..$^{10,11}$ For cancer survivorship, management as a chronic condition would need a comprehensive care package with the input of both primary and secondary care.

The views of patients and healthcare professionals are important in informing policy, especially in the absence of conclusive evidence regarding the comparative value of cancer follow-up models. ${ }^{12}$ Therefore, a systematic review of qualitative studies was undertaken to explore patients' and healthcare professionals' views and preferences with regard to cancer follow-up. This was part of a broader systematic review that also evaluated the effectiveness and cost-effectiveness of primary versus secondary care follow-up of cancer patients, the findings of which are presented separately. ${ }^{13}$ The broader review also looked at nurse-led follow-up and the findings of which are reported elsewhere. ${ }^{14}$

\section{METHOD}

The following databases were searched (from inception to February 2007) using strategies designed specifically for each database: MEDLINE, MEDLINE in process, EMBASE, CINAHL, PsychINFO, AMED, BIOSIS, Index to Scientific and Technical proceedings, Science Citation Index, Social Science Citation Index, Cochrane Central Register of Controlled Trials, Database of Abstracts of Reviews of Effects, Cochrane Database of Systematic Reviews, Health Technology Assessment database, NHS Economic Evaluation database, System for Information on Grey Literature, British Nursing Index, Health Management Information Consortium, National Research Register, and other trial registries $(n=7)$ available via the internet. No language restrictions were used; full details of the search strategy are available on request and described elsewhere ( $\mathrm{R}$ Lewis et al, unpublished data, 2007). (For more information see Appendix 1, Lewis et $a^{/ 13}$ ). Additional references were identified through reviewing the bibliographies of 16 retrieved systematic reviews and 42 included studies, and hand searching five conference proceedings.

The search included qualitative studies that used interviews, focus groups, or open-ended questions to elicit patients' or healthcare professionals' views or preferences regarding cancer follow-up (irrespective of provider). Surveys using only closed questions were excluded. The population of interest included patients of any age who had received treatment for any type and stage of cancer. Only studies that examined follow-up for the following

\section{How this fits in}

Routine hospital follow-up does not lead to early diagnosis of recurrence and improved survival in most cancer sites. However, patients want follow-up to

allay fears of recurrence and provide psychosocial support. There is a need for improved information and education for cancer patients to enable them to make an informed choice about follow-up and to promote their autonomy. In terms of organisation, improved communication between primary and secondary care is essential. Alternative models of follow-up should involve the whole cancer team, including primary care, and include training, support, and rapid access to investigations.

purposes were included: to identify recurrent tumours or new primary disease; to provide support for complications or delayed side-effects of treatments; or to identify patients requiring additional help or treatment (for example, for functional or psychological problems). Studies of patients still receiving hospital-based treatment (for example; radiotherapy), rehabilitation, or specialist palliative care were excluded. However, patients in follow-up who were receiving long-term therapy, such as hormonal treatment for breast or prostate cancer, (but free of active disease) were included.

Review of people's views was carried out using the method reported by Thomas and Harnden, ${ }^{15}$ and Harden et al, ${ }^{16}$ which followed guidelines for thematic analysis of textual data. Two reviewers independently assessed the results of the literature searches and the relevance of retrieved studies. Data pertaining to each study's methodology and context were extracted by one reviewer and checked by a second independent reviewer, while the data were extracted by two independent reviewers. Disagreements were resolved by discussion.

Quality assessment was conducted by two independent reviewers based on the Critical Appraisal Skills Programme ${ }^{17}$ and a checklist developed by Greenhalgh and Taylor. ${ }^{18}$ Disagreements were resolved by discussion. Studies were not excluded from the analysis based on quality.

Key points identified in the results section of the primary studies represented the basic units of the review analysis. Where possible, these were extracted verbatim to avoid misinterpretation. Where results were presented in more lengthy, unfocused prose, the key points were paraphrased succinctly, adhering as closely as possible to the language and meaning of the original. Data reported in the discussion were disregarded, as were direct quotes. The resulting list of key points was then coded by two independent reviewers using QSR N6 software (QSR International), with most points being allocated multiple codes. The final coding was agreed by three 
reviewers. The codes were organised into a hierarchical tree structure and overarching descriptive themes identified using group discussion, in keeping with the methodology reported by Thomas et al. ${ }^{15}$ The study then moved onto the next stage of the analysis that went beyond the findings of the original studies: from peoples' views captured by the descriptive themes, the reviewers inferred positive or negative characteristics for different aspects of follow-up.

The implication of these findings for policy and practice was then considered, in light of the broader aims of the review, the findings of the effectiveness section of the review, the increasing push to discontinue routine hospital follow-up, and the development of alternative models of follow-up. This was initially developed by two independent reviewers and then checked and commented on by two further independent reviewers. The final list of 'ideal world' implications was discussed and agreed by three reviewers, while re-examining the data captured by the descriptive themes.

\section{RESULTS}

The electronic searches identified 43861 references of which 232 papers were retrieved in full. Thirty-one additional studies were identified by hand searching (see Figure 1, Lewis et $a l^{13}$ ).

Nineteen relevant qualitative studies published in full were identified. ${ }^{1,12,19-35}$ Two further studies that were only published as conference abstracts were identified, and are not discussed further due to the limited information presented. ${ }^{36,37}$ Two projects on the National Research Register were identified, for which no results were available. ${ }^{38,39}$

Eight studies were linked to randomised controlled trials (RCTs) evaluating different modes of follow-up (included in the effectiveness section of this systematic review). ${ }^{19,21,22,24,27-29,31}$ Two were related to the same trial and examined the intervention and control groups separately. ${ }^{28,29}$

A summary of included studies is presented in Table 1. Ten studies evaluated breast cancer followup, ${ }^{1,19,20,22,24,26,28-30,32}$ four multiple cancer sites, ${ }^{21,27,34,35}$ two colorectal, ${ }^{12,33}$ two lung, ${ }^{25,31}$ and one study evaluated gynaecological cancers. ${ }^{23}$ Eight studies examined the views of healthcare professionals, ${ }^{1,21,25-27,30,31,35}$ four of which examined the views of GPs..$^{21,25-27}$ Sixteen studies included patients; ${ }^{1,12,19-26,28-30,32-34} 15$ collected patients' views on routine hospital follow-

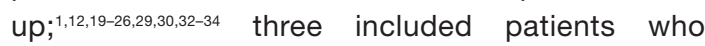
experienced nurse-led follow-up,,$^{24,28,30}$ and two included patients who received GP follow-up. ${ }^{12,21}$ The number of included patients ranged from $\mathrm{six}^{20}$ to $113^{19}$ (median $\left.=24\right)$, and healthcare professionals from two $^{31}$ to $120^{1}$ (median $=7$ ). The number of included participants was not stated for one study. ${ }^{26}$ Most studies were European (12 undertaken in the UK, $, 112,19,20,22-26,30-32$ two in Norway, ${ }^{21,27}$ and two in Sweden ${ }^{28,29}$ ). Two were carried out in $\mathrm{Canada}^{33,35}$ and one in China (Hong Kong). ${ }^{34}$ The time period over which the data were collected was not stated for most studies; where it was reported (six studies) most of these studies were undertaken within the time period 2000-2005, 12,19,21,25,30 with one study collecting data between 1992 and 1993. ${ }^{35}$ Various data-collection methods were used, with some studies using more than one method, which included direct observation of consultation, ${ }^{1}$ focus groups ${ }^{22,26,34}$ in-depth interviews, ${ }^{12,20,25}$ semi-structured interviews, ${ }^{1,19,21,23,27-33,35}$ structured interviews, ${ }^{24}$ and survey questionnaires (with open-ended questions). ${ }^{1,21,25}$

Study quality is presented in Table 2. Most studies were poorly reported and the quality of the methods could not be assessed. In general, the sample strategy and size, and reasons why some participants declined to participate were poorly described or not reported. Summary characteristics of included participants were missing in many studies. The researchers' perspectives could have influenced responses in some studies (for example, nurse investigating nurse-led follow-up), and was often not reported. Few studies reported testing the credibility of their findings; it was not clear if all the data had been taken into account and whether negative or discrepant findings were addressed. When reporting conclusions, few studies explored alternative explanations or discussed the limitations of their findings.

\section{Findings of included studies}

Twelve descriptive themes were identified; these were interpreted to develop a list of 12 recommendations for policy and practice (Box 1).

Fear of recurrence was the main reason for patients' anxiety and need for reassurance. Patients feared recurrence, especially during the early phase after completing treatment, ${ }^{1,22,23,32,34}$ and wanted regular surveillance. ${ }^{22}$ Healthcare professionals' reluctance to talk about 'cure', and patients' understanding of 'remission' as a stage in the disease rather than a period of good health, helped to maintain the focus on detection of recurrence. ${ }^{23,26}$

Conventional follow-up, although intended to allay anxiety, exacerbated patients' need for reassurance. A system of regular check-ups with tests and examinations provided only temporary reassurance; and by the time of the patients' next appointment their anxiety had returned. ${ }^{1,12,20,22,23,25,26,29,32}$ Healthcare 


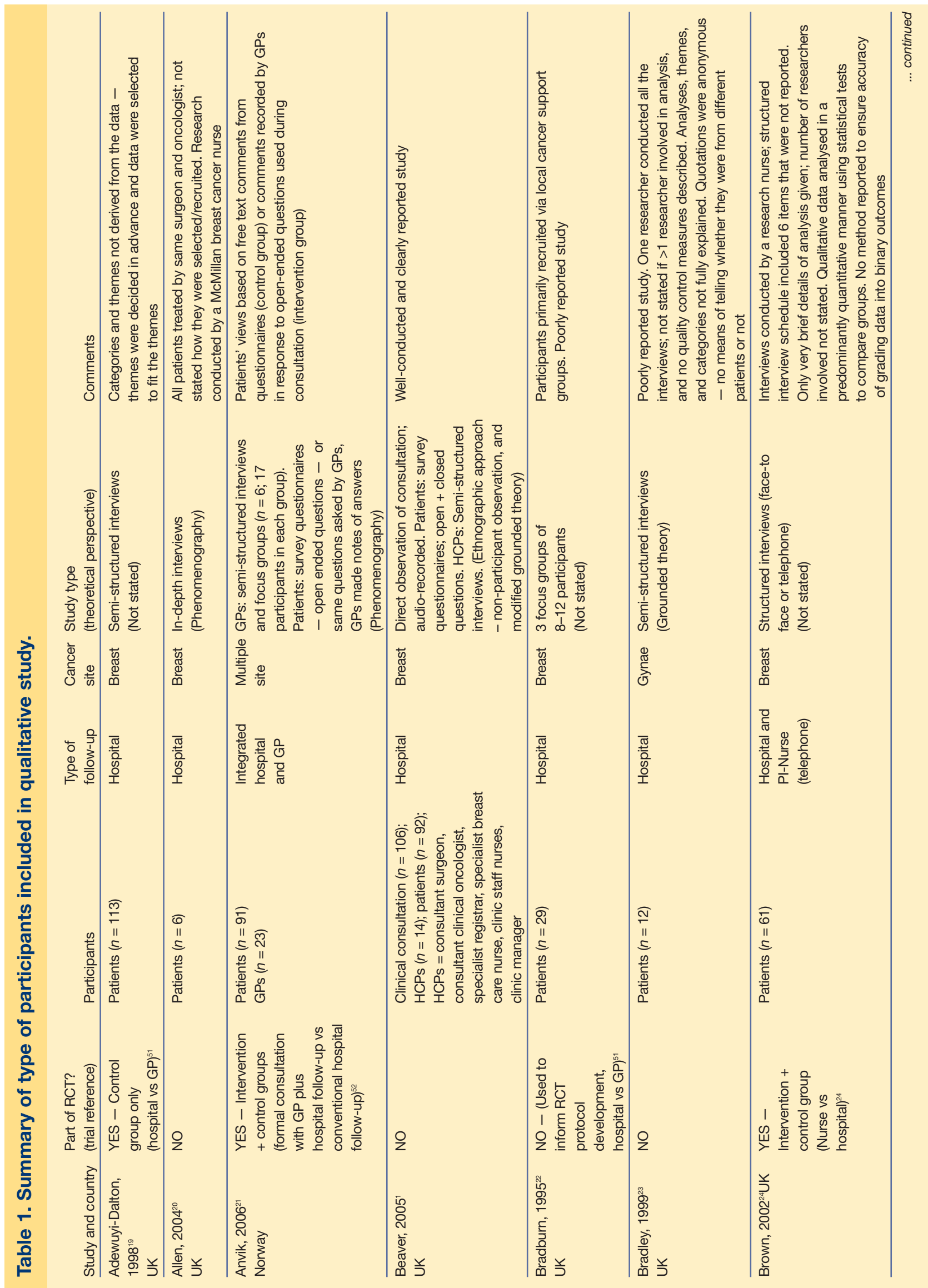




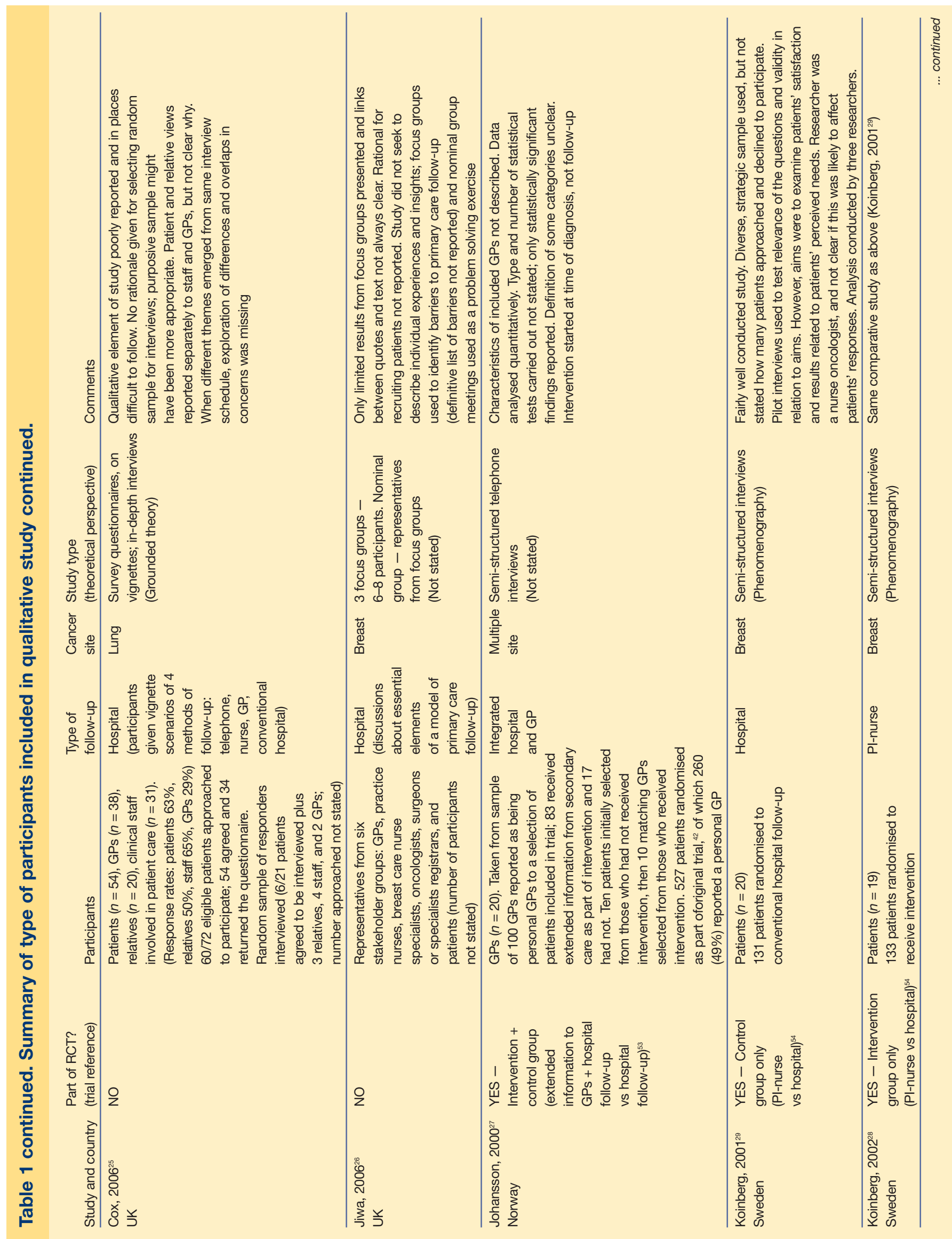




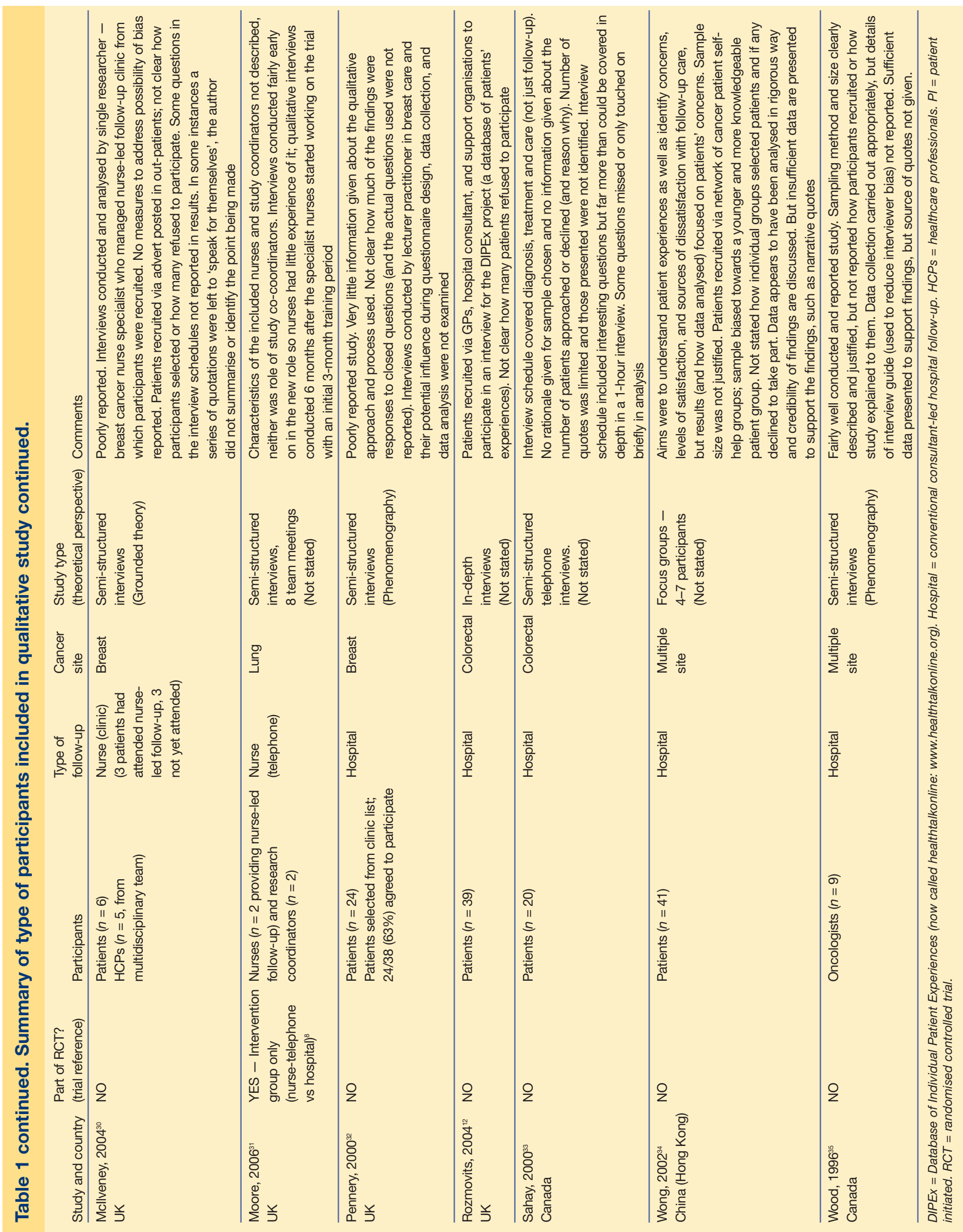




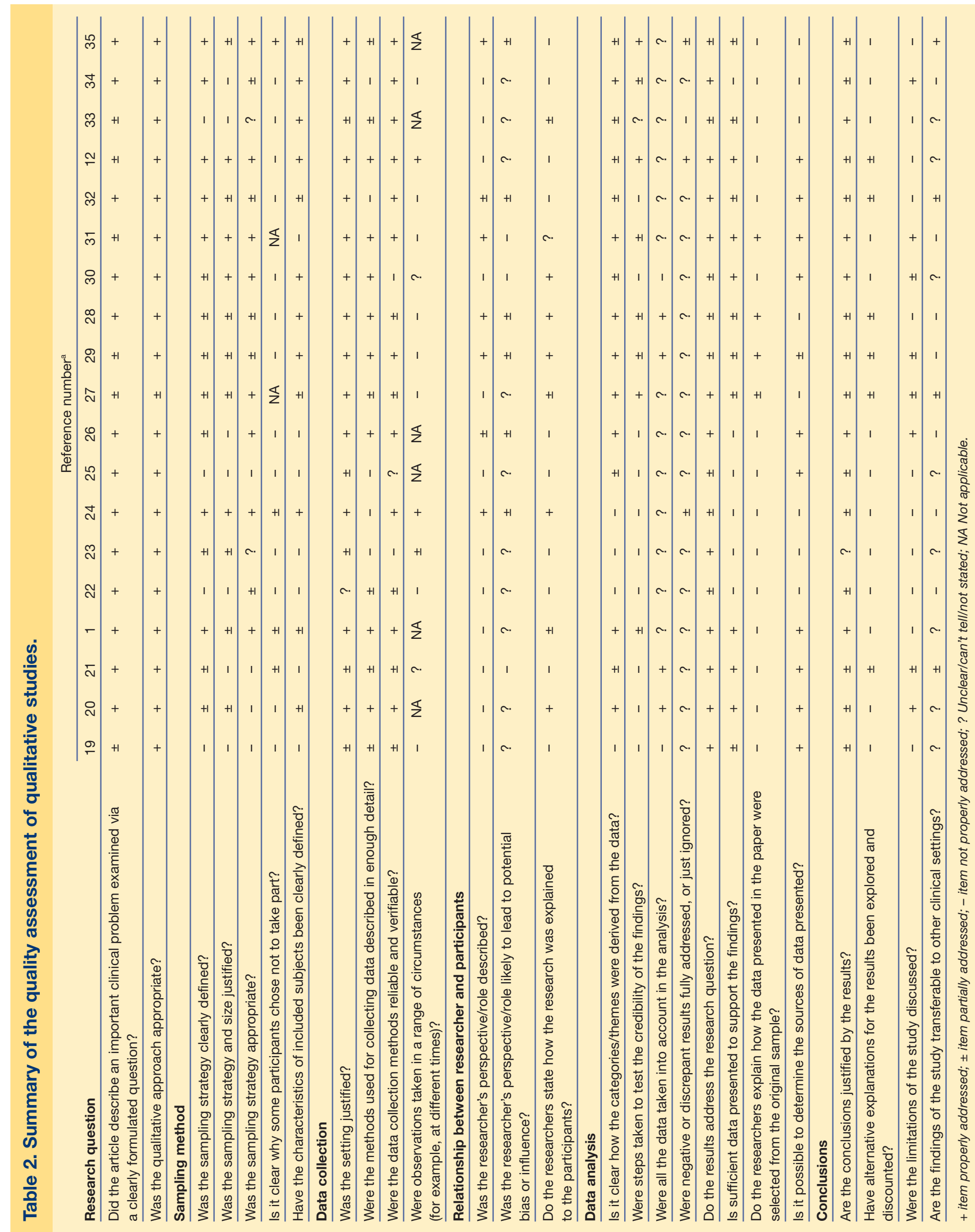


professionals felt that patients expected tests and examinations, and were difficult to reassure without them. ${ }^{1,26}$ For some patients an increase in the interval between appointments was seen as a positive sign, ${ }^{1,32}$ and anxiety diminished over time, ${ }^{23}$ but, for others, anxiety persisted ${ }^{20,23,26}$ and even increased as the interval lengthened. ${ }^{1,23}$

Specialist knowledge and quick access to tests were thought to be the most important ingredients of follow-up, and were key concepts of alternative models. Access to expertise, including a team of specialists with associated technology, engendered confidence in patients and relatives and was the main advantage of hospital-based follow-up..$^{19,22,25,32}$ Patients' antagonism towards alternative models was largely associated with concerns that it would be difficult to re-access specialist facilities quickly if required. $\underline{\text { 22 }}$ GPs were considered by patients, ${ }^{12,19,23,32,33}$ and oncologists ${ }^{35}$ to lack specialist knowledge for undertaking follow-up, identifying and treating recurrence, or even supporting patients between hospital appointments. However, GPs thought this was not a barrier to involvement as they could get information from specialists when needed..$^{21}$ Cancer specialist nurses were seen to be less knowledgeable and experienced than doctors, but had quick access to medical back up when necessary. ${ }^{25,26}$

Information regarding the effectiveness of follow-up (tests and examinations) was not given to patients. Healthcare professionals admitted that patients were given little or no explanation of follow-up and thought patients would be more likely to accept alternative models if they were fully explained. ${ }^{25,26}$ Patients were unaware of flaws in conventional hospital follow-up. Inconsistencies in surveillance protocols between doctors and hospitals confused and worried them. ${ }^{22,34}$ Tests and examinations appeared to provide tangible evidence of freedom from disease, 12,19,23,25,29 and radiological tests were considered to be particularly reliable by patients. ${ }^{20,23,25}$ However, healthcare professionals acknowledged that examinations were unlikely to detect recurrence in patients who were symptom-free. ${ }^{1,26}$

Patients lacked clear information that could aid coping and enable involvement. Patients obtained information from various sources (friends and family, ${ }^{33}$ internet and support groups, ${ }^{12,33} \mathrm{GPs},{ }^{33}$ and hospital ${ }^{1,33,34}$ ), but still had unmet needs. ${ }^{12,19,21,25,28,29,32-34}$ Appropriate information reduced anxiety, ${ }^{23}$ provided reassurance, ${ }^{23}$ helped them cope, ${ }^{23,34}$ and enabled involvement. ${ }^{33,34}$ However, many were dissatisfied with the limited information given in
Box 1. Recommendations for policy and practice derived from descriptive themes.

1. Patients should be given full and clear information using plain language on the following:

- effectiveness of different tests and examinations in detecting recurrence;

- risk of recurrence and what they can do to reduce this;

- potential side-effects of treatment and how to deal with them;

- signs and symptoms of potential recurrence and what to do if these are experienced; and

- alternative models of follow-up that may be available.

2. A follow-up care plan which has been negotiated with the patient/carer should be set up for each patient on completing treatment, including the patient's preferred model of follow-up. Generally, patients' main concern is fear of recurrence and many find regular follow-up reassuring.

3. Healthcare professionals should provide sufficient time and encourage patients during follow-up to raise questions and concerns.

4. Psychological support should be an integral part of follow-up, especially during the initial stages.

5. Tests and examinations should not be conducted purely for reassurance, but only where there is a reason or the evidence base to support their use; and this must be explained to the patient/carer.

6. Patients should be given contact details of a key person whom they can contact when needed and who can provide them with support and continuity of care.

7. Multidisciplinary teams should include representation from primary care (but this does not have to be the individual patient's GP or someone from the patient's practice)

8. There needs to be a formal handover and exchange of information between primary and secondary care. This should include complete discharge information and exchange of contact details (hospital clinicians to GPs and vice versa).

9. As an adjunct to routine hospital follow-up, a member of the primary care team should make contact with the patient immediately after hospital discharge to discuss the type of support that primary care could offer.

10.Patients should be given informed choice about whether to attend scheduled appointments or just when they have problems or symptoms (patient-initiated).

11.If alternative models of follow-up (for example, primary care, nurse, or patient-initiated follow-up) are to be developed and tested in further research, then these models should:

- include a system of rapid referral for investigations (to be explained to patients);

- include training/education for GPs, nurses, and other healthcare professionals;

- include support from specialist team at hospital (or medical support for alternative models set up in secondary care);

- be established with the collaboration of the whole cancer team and primary care from the outset; clear protocol/guidelines should be agreed by all parties in advance; and

- enable individual GPs (for primary care follow-up) to be able to opt out; alternative primary care-based follow-up should be provided if the patient wishes this.

12.The role of cancer support groups needs to be explored further. 
consultations, ${ }^{12,19,21,25,28,29,32-34}$ or felt advice was inappropriate. ${ }^{12}$ Patients had difficulty understanding medical terminology and thought technical language was sometimes used as an excuse not to provide adequate information. ${ }^{33,34}$ Clinic nurses interpreted information for patients. ${ }^{1}$ Some patients felt inhibited about asking questions. ${ }^{32,33}$ Some patients perceived that more information was given to patients who were better educated, ${ }^{34}$ or asked the right questions. $^{33,34}$

Continuity of care and unhurried consultations were of major importance to patients. A good relationship with the healthcare professional was valued by patients, and continuity of care (seeing the same person) was key to this. ${ }^{19-21,25,29,30,32,34}$ Patients appreciated being seen by someone familiar who knew their case. ${ }^{12,32}$ Having contact details of healthcare professionals was considered important and reassuring. ${ }^{12,25,32}$ Lack of continuity was a barrier to good communication..$^{30,34}$ Patients found that nurses were easier to talk to than doctors and had more time. ${ }^{25,30}$ The system of rotating junior doctors interrupted continuity, ${ }^{34}$ and there was no time during consultations to raise concerns or questions..$^{1,19-21,32,33}$

Psychosocial support was important because of the impact of cancer on patients' lives (for example, social, domestic, economic) but was under-provided. Psychosocial support was very important to patients, ${ }^{20,22,23,28,29,33}$ but patients and healthcare professionals agreed that there was not enough time for it in conventional follow-up. ${ }^{1,20,32}$ Some support could be provided by family and friends,,$^{20,23,29}$ although some people were reluctant to burden them. ${ }^{33}$ Some found GPs helpful, 12,33 although others lacked confidence in them to provide support.22 Specialist nurses had more time for psychosocial support than doctors, and were particularly effective in providing it. ${ }^{12,25,31,32}$

Patients were reluctant to use their GP for cancerrelated support (in between hospital visits). While a few GPs were keen to participate in cancer care,,$^{21}$ and some patients received valuable support, ${ }^{12,33}$ many patients thought GPs were too busy, ${ }^{22,32}$ lacked knowledge, ${ }^{23,25,33}$ or were not interested in cancer care. ${ }^{12,22}$ Some GPs were understanding and helpful, but patient-GP relationships could be undermined by problems during diagnosis and initial referral, ${ }^{12}$ or by lack of rapport. ${ }^{22}$

There were significant communication problems, in both directions, between primary and secondary care, which hindered GPs' ability to provide support. Multidisciplinary teams were thought to be very important and to work well in the hospital setting, , $^{25,35}$ but the absence of a primary care representative was thought a weakness. ${ }^{35}$ GPs felt uninformed, and a formal handover with exchange of information at the time of patients' discharge from hospital was identified (by GPs and hospital doctors) as most important. ${ }^{21,26,27,35}$ In an RCT, GPs who were given more information by the hospital felt more able to be involved in follow-up, to determine patients' needs, and to offer support. ${ }^{27}$ Oncologists felt that they were not kept informed by GPs and thought the large number of GPs, their varied level of commitment and knowledge, lack of time, and difficulties in contacting them were barriers to collaboration between specialists and primary care ${ }^{35}$ Patients' preferences for either hospital or GP follow-up were also seen to be detrimental to collaboration and a team approach. ${ }^{35}$

Cancer specialist nurse-led follow-up could benefit patients but some healthcare professionals lacked confidence in it. Patients had confidence in nurse-led follow-up, and women especially were thought to be more comfortable with female nurses, but some healthcare professionals did not think nurses were experienced enough. ${ }^{30}$ A newly set-up nurse-led telephone follow-up service was seen by specialist nurses to provide effectively planned and managed care in a more supportive environment that allowed more involvement of the family in care. ${ }^{32}$ However, the nurses experienced hostility and lack of support from hospital staff, and it took time for them to become confident in their role and to earn the respect of other colleagues. ${ }^{31}$

Some thought that nurse-led follow-up was inappropriate and that nurses were not skilled enough to deal with the medical interventions and palliative care required by lung cancer patients. ${ }^{31}$ Nurses reported that the role carried an enormous emotional burden and that they needed supervision and support, but that it was immensely satisfying. ${ }^{31}$ Clinic staff thought that nurse-led follow-up for lung cancer could free up doctors' time for patients with medical needs, while patients thought it could give access to expertise more quickly than conventional follow-up..$^{25}$ Clinical staff identified the essential requirements for nurse-led follow-up (for lung cancer patients) as: clear protocol and guidelines, access to medical back-up, and training. ${ }^{25}$

GPs were not thought to be willing or to have sufficient expertise to conduct primary care followup. Patients lacked confidence in GPs to provide follow-up, and those attending conventional hospital follow-up chose GP-led follow-up as their least preferred option from four models (nurse, 
conventional, telephone, and GP follow-up). ${ }^{22,25}$ Patients thought GPs would be unwilling to provide follow-up, ${ }^{12,22}$ and healthcare professionals thought they would need additional training first. ${ }^{26}$ Key stakeholders thought that the essential requirements for primary-care follow-up (for breast cancer patients) included formal handover from secondary care with a detailed case summary, a protocol for rereferral to specialists, access to investigations, and referral to a specialist counsellor. ${ }^{26,27}$ There was no consensus on whether patients should have regular follow-up appointments or make an appointment when symptoms or concerns arose. ${ }^{26,29}$

Patient-initiated follow-up was convenient but less reassuring. Some patients preferred the flexibility of patient-initiated follow-up, but others preferred regular appointments, ${ }^{29,32}$ in some cases because they feared they would put off or fail to make an appointment. ${ }^{23,32}$

\section{DISCUSSION}

\section{Summary of main findings}

Twelve descriptive themes were identified. Fear of recurrence was the main concern for patients, which was temporarily alleviated by attending routine follow-up with cancer specialists. Information regarding the limitations of routine hospital follow-up (or lack of effectiveness of examinations and tests) was not conveyed to patients, who also had unmet needs for information, which would help them cope and be more involved.

Continuity of care, unhurried consultation, and psychosocial support were important to patients. The expertise of hospital specialists and quick access to tests were highly valued, but time, emotional support, and continuity of care were sometimes lacking in routine hospital follow-up. Patients were reluctant to use their GP for cancerrelated support in between hospital visits, and GPs were thought to be unwilling or to have insufficient time and expertise to provide follow-up. There were significant communication problems between primary and secondary care, in both directions. Specialist nurses were considered more supportive than doctors, and patients were satisfied with nurseled follow-up but other health professionals lacked confidence in it. Patient-initiated follow-up was more convenient but less reassuring.

From the descriptive themes, 12 perceived implications for policy and practice were derived. Improved patient information is needed, which would help them to cope and be more involved as well as enable them to make informed choices about the type of follow-up support they need. Patients valued regular follow-up by specialists to allay fears of disease recurrence. Psychological support should be an integral part of follow-up, and sufficient time and encouragement should be given for patients to raise concerns and ask questions. Improved communication between primary and secondary care should be established from the outset, with a formal handover and exchange of information at discharge. The availability and type of primary care support (as an adjunct to routine hospital follow-up) should be conveyed to patients. Alternative models of follow-up should be established with the support of the whole cancer team (including input from primary care), and include training and educational support, and quick access to specialist tests.

\section{Strengths and limitations of the study}

The literature search was comprehensive, but it was carried out separately for each cancer site, rather than using generic cancer terms. Although the searches identified studies evaluating multiple cancer sites, it is not possible to be certain that none was missed, especially studies examining cancer survivorship among children or young adults, which did not specify the cancer site. The quality of included studies varied, with many being poorly reported. However, there is debate about the use of quality-assessment tools for appraising qualitative studies. ${ }^{40-42}$ The included studies were heterogeneous in terms of population studied, underlying theory used, and methods of data collection. Although there is some controversy about the value of combining the findings from studies using different methods or informed by different theories of knowledge, ${ }^{43,44}$ this enabled the researchers to identify a range of common themes.

Most included studies were related to conventional follow-up in secondary care, which was the type of follow-up that most had received. Some of the views expressed concerning other models of care were based on participants' ideas, rather than their experiences. The search only included qualitative studies rather than quantitative surveys of participants' views, because the views expressed were more likely to reflect those of the participants rather than any perceived views or beliefs of the researchers. It must be acknowledged that there is a minor limitation that the potential prevalence of these views and themes was not estimated.

The recommendations for policy are based on an evaluation that goes beyond the findings of the primary studies, and as such their development was dependent on the judgement and insights of the reviewers and their knowledge of relevant literature from different sources. They were generated using the same underlying rigorous process as the inductive analysis and development of the 
descriptive themes; accordingly, they are presented within the results rather than the discussion. The recommendations were initially developed by two independent reviewers who are not clinicians. They were then checked and commented on by two further independent reviewers, one of whom is a practising GP and the second a consultant oncologist.

\section{Comparison with existing literature}

The authors are not aware of any other systematic reviews that summarise the findings of peoples' views on cancer follow-up.

\section{Implications for future research and clinical practice}

Patients wanted regular consultations with cancer specialists, tests, and examinations to allay fears of disease recurrence. Healthcare professionals admitted that patients were given little or no explanation about follow-up, and patients felt that appropriate information could help them to cope and would enable involvement.

The evidence regarding the effectiveness of followup should be discussed with patients, as well as their own personal risk of recurrence including advice on what they can do, if anything, to reduce this risk (for example, there is evidence that exercise and a reduced-fat diet can reduce the risk of breast cancer recurrence ${ }^{45-47}$ ). In a survey of 156 colorectal cancer patients attending routine hospital follow-up, only $22 \%$ could identify risk indicators for recurrence, while $64 \%$ said they would like to be told what to look for. ${ }^{48}$

Patients may also benefit from education and information on how to cope psychologically, and self-management strategies for common problems. The opportunities for patients to participate actively in their own post-treatment care were not discussed in any of the primary studies, nor how such opportunities could be built into any model of cancer follow-up.

To enable comprehensive patient care and for patients to have the support they need, improved communication between primary and secondary care is needed in both directions, irrespective of the type of follow-up used. National Institute for Health and Clinical Excellence guidance on breast and colorectal cancer recommends that the multidisciplinary team responsible for the patient should maintain close contact with GPs and primary care teams, ${ }^{49,50}$ and should take responsibility for passing clinical information in a timely manner to the GP, irrespective of the type of follow-up. Having a GP with a special interest in cancer as a member of the multidisciplinary team, who could act as a representative or spokesperson for primary care, could be beneficial.

Most of the qualitative studies included patients who had not experienced alternative types of followup; they were mainly patients who had recently completed treatment or who had received conventional hospital follow-up. More qualitative studies are needed of patients who have experienced other forms of follow-up. Further research specifically focusing on preferences and experiences (as opposed to views and attitudes) would also be welcomed in this topic. More qualitative studies are also needed of cancer sites not covered by included studies.

Further research is needed to evaluate whether patients' preference for routine follow-up is changed if they are given clear information about the effectiveness of diagnostic tests and investigations, their risk of recurrence, how to recognise signs and symptoms of recurrence, and any preventative measures they could take. Patients value the psychological support provided by routine hospital follow-up. Further research is needed to assess the type of psychological support that patients want or need, and how best to provide this within the context of alternative models of follow-up.

\section{Funding body}

Cancer Research UK (Ref C8350/A4543). The funding source had no involvement in the study

\section{Ethical approval}

Ethical approval was not required

\section{Competing interests}

The authors have stated that there are none

Acknowledgments

We would like to thank Stephen Duffy for his advice on searching databases and developing the search strategy, Bernadette Coles and Hilary Kitcher for checking some of the original search strategies, Madeline Pasterfield and Jennifer Wilkinson for technical support including carrying out the update searches, and Alison Eastwood for peer reviewing the protocol and draft final report. We would also like to thank the following people for sending us further information about the studies they had conducted: Kinta Beaver, Gillian Knowles, Karen Cox, Christine Mcllvenney, and Stephen Barclay. Thanks also to Richard Bailey and his staff at the John Spalding Library for their help in retrieving manuscripts.

\section{Discuss this article}

Contribute and read comments about this article on the Discussion Forum: http://www.rcgp.org.uk/bjgp-discuss

\section{REFERENCES}

1. Beaver K, Luker K. Follow-up in breast cancer clinics: reassuring for patients rather than detecting recurrence. Psychooncology 2005; 14(2): 94-101.

2. Rojas MP, Telaro E, Russo A, et al. Follow-up strategies for women treated for early breast cancer. Cochrane Database Syst Rev 2000; (4): CD001768.

3. Somerfield MR, Schapira DV, Davidson NE, et al. Recommended breast cancer surveillance guidelines. J Clin Oncol 1997; 15(5): 2149-2156.

4. Collins RF, Bekker HL, Dodwell DJ. Follow-up care of patients treated for breast cancer: a structured review. Cancer Treat Rev 2004; 30(1): 19-35.

5. Botteman MF, Pashos CL, Redaelli A, et al. The health economics of 
bladder cancer: a comprehensive review of the published literature. Pharmacoeconomics 2003; 21(18): 1315-1330.

6. Francken AB, Bastiaannet E, Hoekstra HJ. Follow-up in patients with localised primary cutaneous melanoma. Lancet Oncol 2005; 6(8): 608-621.

7. Pascoe SW, Neal RD, Allgar VL, et al. Psychosocial care for cancer patients in primary care - recognition of opportunities for cancer care. Fam Pract 2004; 21(4): 437-442.

8. Moore S, Corner J, Haviland J, et al. Nurse led follow up and conventional medical follow up in management of patients with lung cancer: randomised trial. BMJ 2002; 325(7373): 1145-1147.

9. Ham C. Gordon Brown's agenda for the NHS [editorial]. BMJ 2008; 336(7935): 53-54.

10. Campbell R, Pound P, Pope C, et al. Evaluating meta-ethnography: a synthesis of qualitative research on lay experiences of diabetes and diabetes care. Soc Sci Med 2003; 56(4): 671-684

11. Griffin SJ, Kinmonth AJ, Skinner C, Kelly J. Education and psychosocial interventions for adults with diabetes. London: British Diabetic Association, 1999.

12. Rozmovits L, Rose P, Ziebland S. In the absence of evidence, who chooses? A qualitative study of patients' needs after treatment for colorectal cancer. J Health Serv Res Policy 2004; 9(3): 159-164.

13. Lewis RA, Neal RD, Williams NH, et al. Follow-up of cancer in primary care versus secondary care: systematic review. Br J Gen Pract 2009; 59: 525-532.

14. Lewis R, Neal RD, Williams NH, et al. Nurse-led vs conventional physician-led follow-up for patients with cancer: systematic review. $J$ Adv Nurs 2009; 65: 706-723.

15. Thomas J, Harden A. Methods for the thematic synthesis of qualitative research in systematic reviews. BMC Med Res Methodol 2008; 8: 45. DOI: 10.1186/1471-2288-8-45

16. Harden A, Garcia J, Oliver S, et al. Applying systematic review methods to studies of people's views: an example from public health research. J Epidemiol Community Health 2004; 58(9): 794-800.

17. Critical Appraisal Skills Programme (CASP). Critical Appraisal Skills Programme (CASP) making sense of evidence. Oxford: Public Health Resource Unit (PHRU), 2006.

http://www.phru.nhs.uk/Doc_Links/Qualitative\%20Appraisal\%20Too .pd

18. Greenhalgh T, Taylor R. How to read a paper: papers that go beyond numbers (qualitative research). BMJ 1997; 315(7110): 740-743.

19. Adewuyi-Dalton R, Ziebland S, Grunfeld E, Hall A. Patients' views of routine hospital follow-up: a qualitative study of women with breast cancer in remission. Psychooncology 1998; 7(5): 436-439.

20. Allen A. The meaning of the breast cancer follow-up experience for the women who attend. Eur I Oncol Nurs 2002; 6(3): 155-161.

21. Anvik T, Holtedahl KA, Mikalsen H. 'When patients have cancer, they stop seeing me' - the role of the general practitioner in early followup of patients with cancer - a qualitative study. BMC Fam Pract 2006; 7: 19

22. Bradburn J, Maher J, Adewuyidalton R, et al. Developing clinical-tria protocols - the use of patient focus groups. Psychooncology 1995; 4(2): 107-112.

23. Bradley EJ, Pitts M, Redman CWE, Calvert E. The experience of longterm hospital follow-up for women who have suffered early stage gynecological cancer: a qualitative interview study. Int J Gynecol Cancer 1999; 9(6): 491-496.

24. Brown L, Payne S, Royle G. Patient initiated follow up of breast cancer. Psychooncology 2002; 11(4): 346-355.

25. Cox K, Wilson E, Heath W, et al. Preferences for follow-up after treatment for lung cancer. Cancer Nurs 2006; 29(3): 176-187.

26. Jiwa M, Thompson J, Coleman R, Reed M. Breast cancer follow-up: could primary care be the right venue? Curr Med Res Opin 2006; 22(4): 625-630.

27. Johansson B, Berglund G, Hoffman K, et al. The role of the genera practitioner in cancer care and the effect of an extended information routine. Scand J Prim Health Care 2000; 18(3): 143-148.

28. Koinberg IL, Holmberg L, Fridlund B. Breast cancer patients' satisfaction with a spontaneous system of check-up visits to a specialist nurse. Scand J Caring Sci 2002; 16(3): 209-215.

29. Koinberg I, Holmberg L, Fridlund B. Satisfaction with routine followup visits to the physician - the needs of patients with breast cancer. Acta Oncol 2001; 40(4): 454-459.

30. McIlveney C. Evaluation of the nurse led follow up clinic for breast cancer patients. Middlesbrough: University of Teeside, 2004.
31. Moore S, Wells M, Plant $\mathrm{H}$, et al. Nurse specialist led follow-up in lung cancer: the experience of developing and delivering a new model of care. Eur J Oncol Nurs 2006; 10(5): 364-377.

32. Pennery E, Mallet J. A preliminary study of patients' perceptions of routine follow-up after treatment for breast cancer. Eur J Oncol Nurs 2000; 4(3): 138-145.

33. Sahay TB, Gray RE, Fitch M. A qualitative study of patient perspectives on colorectal cancer. Cancer Pract 2000; 8(1):38-44.

34. Wong DKP, Chow SF. A qualitative study of patient satisfaction with follow-up cancer care: the case of Hong Kong. Patient Educ Couns 2002; 47(1): 13-21.

35. Wood ML, McWilliam CL. Cancer in remission. Challenge in collaboration for general practitioners and oncologists. Can Fam Physician 1996; 42: 899-904; 907-910.

36. Hanselius P. Women operated for breast cancer - their opinion of follow-up by breast cancer nurse [poster 1628]. Eur J Cancer Suppl 2005; 3(2): 470.

37. Murchie P, Hannaford PC, Nicolson MC, et al. Attending the general practitioner for routine melanoma follow-up: the practical experiences of participants in an RCT. In: The Society for Academic Primary Care 35th Annual Scientific Meeting; 2006 12-14 July 2006, Keele University, UK.

38. Hughes DC. Breast cancer follow-up; a focus group study to explore women's experiences and preferences in relation to type and venue of follow-up [Abstract]. National Research Register (NRR) (accessed Feb 2007).

39. Thompson J. Breast cancer after care: psycho-social support in general practice [Abstract]. National Research Register (NRR) (accessed Feb 2007).

40. Dixon-Woods M, Bonas S, Booth A, et al. How can systematic reviews incorporate qualitative research? A critical perspective. Qual Res 2006; 6: $27-44$

41. Mays N, Pope C. Qualitative research in health care: assessing quality in qualitative research. BMJ 2000; 320(7226): 50-52.

42. Spencer L, Ritchie J, Lewis J, Dillon L, National Centre for Social Research. Quality in qualitative evaluation: a framework for assessing research evidence. London: Government Chief Social Researcher's Office, 2003

43. Pope C, Mays N, Popay J. Synthesizing qualitative and quantitative health evidence: a guide to methods. Maidenhead: McGraw Hill, 2007

44. Dixon-Woods M, Agarwal S, Young B, et al. Integrative approaches to qualitative and quantitative evidence. London: Health Development Agency, 2004.

45. Chlebowski RT, Blackburn GL, Elashoff RE, et al. Dietary fat reduction in postmenopausal women with primary breast cancer: Phase III Women's Intervention Nutrition Study (WINS). J Clin Onco 2005; 23(16S): 10.

46. Holmes MD, Chen WY, Feskanich D, et al. Physical activity and survival after breast cancer diagnosis. JAMA 2005; 293(20) 2479-2486.

47. Patel AV, Press MF, Meeske K, et al. Lifetime recreational exercise activity and risk of breast carcinoma in situ. Cancer 2003; 98(10): 2161-2169.

48. Papagrigoriadis S, Heyman B. Patients' views on follow up of colorectal cancer: implications for risk communication and decision making. Postgrad Med J 2003; 79(933): 403-407.

49. National Institute for Clinical Excellence. Improving outcomes in colorectal cancers - the manual update. London: National Institute of Clinical Excellence, 2004.

50. National Institute for Clinical Excellence. Improving outcomes in breast cancer - the manual update. London: National Institute for Clinical Excellence, 2002

51. Grunfeld E, Mant D, Yudkin P, et al. Routine follow up of breas cancer in primary care: randomised trial. BMJ 1996; 313(7058): 665-669.

52. Holtedahl K, Norum J, Anvik T, Richardsen E. Do cancer patients benefit from short-term contact with a general practitioner following cancer treatment? A randomised controlled study. Support Care Cancer 2005; 13(11): 949-956.

53. Johansson B, Holmberg L, Berglund G, et al. Reduced utilisation of specialist care among elderly cancer patients: a randomised study of a primary healthcare intervention. Eur J Cancer 2001; 37(17): 2161-2168.

54. Koinberg IL, Fridlund B, Engholm GB, Holmberg L. Nurse-led follow-up on demand or by a physician after breast cancer surgery: randomised study. Eur J Oncol Nurs 2004; 8(2): 109-117. 